\title{
Reaction-diffusion chip implementing analog cellular-automaton model
}

Takashi Yamada, Yoshimasa Honma, Tetsuya Asai, Yoshihito Amemiya

Department of Electrical Engineering, Hokkaido University, Kita 13, Nishi 8, Kita-ku, Sapporo, 060-8628, Japan.

Phone: +81-11-706-7149, Fax: +81-11-706-7890, E-mail: yamada@ sapiens-ei.eng.hokudai.ac.jp

\section{INTRODUCTION}

In GHz-clock speed LSI, clock skew and phase-lagged stable synchronous patterns called modelock are generated using random process variations and RC delays in the clock line [1]. Once this phenomenon occurs, a system will fail because clock-distribution circuits cannot operate in sync. It takes a long time to simulate the phenomenon because of a large number of calculations for nonlinear partial differential equations. Similar phenomena that produce a variety of orders, rhythms, and self-organizing are often observed in nature. A reactiondiffusion (RD) system is a lively, dynamic system that produces such phenomena [2]. Our purpose was to make a hardware accelerator to calculate the modelock phenomenon at a high speed. We describe an RD chip based on an analog cellular-automaton model, which represents our first step toward this goal.

\section{DESCRIPTION OF CHIP}

An RD chip was constructed to imitate the BelousovZhabotinsky (BZ) reaction, a well-known RD system that creates some of the natural rhythms and patterns. One wellknown model that accounts for the BZ reaction is the Oregonator [2]. The transient responses of its two principal variables $(U$ and $V)$ are shown in Fig. 1 with their typical parameter values. Based on these values, the Oregonator exhibits oscillatory or excitatory behavior that is activated only when external stimuli are added.

We proposed a simplified model of the BZ system that uses the cellular-automaton (CA) method. A cellular automaton is a parallel-data processing system consisting of many identical processing elements (cells) arrayed uniformly on a plane (Fig. 2). Each cell changes its state based on its current one and the current state of the neighboring six cells. To simplify the Oregonator model, we introduced a discrete system variable into the CA model even though the Oregonator is normally described using continuous system variables. Three circulative periods were introduced that reflect the values of $U$ and $V$ : active, inactive, and refractory (Fig. 3). In this model, $U$ takes a binary value representing the active $(U=1)$ or inactive period ( $U=0$ ) of the cell, while $V$ takes continuous values representing the degree of inhibition to $U$.

In order to analyze the analysis of the modelock phenomenon using CA, two different operations are carried out to cells according to a system to analyze. That is, the clockdistribution circuit is transposed to the cell that operates in oscillatory mode, and the clock line is transposed to the cell that operates in excitatory mode.

\section{TRANSITION RULES}

In the proposed model, each cell changes its $U$ and $V$ based on transition rules. To implement both oscillatory and exci- tatory behavior, we introduced a mode-select signal that determines the mode, oscillatory or excitatory, in which the cell operates. We defined six transition rules:

1. $V$ increases at a constant rate when the cell is active $(U=1)$.

2. $V$ decreases at a constant rate when the cell is inactive $(U=0)$.

3. $U$ changes from active to inactive (from 1 to 0 ) when $V$ reaches its threshold $\left(V_{\max }\right)$.

4. $U$ changes from inactive to active (from 0 to 1 ) when $V=0$ in oscillatory mode.

5. $U$ changes from inactive to active (from 0 to 1 ) when $V=0$ and more than one neighboring cell is active $(U=$ 1 ) in excitatory mode.

6. At $U=0$ and $V=V r, U$ turns active when more than one neighboring cell is active in oscillatory mode and when more than two neighboring cells are active in excitatory mode.

The last rule indicates that a cell cannot turn active $(U=1)$ when the degree of inhibition $V$ is high and that the cell can turn active to overcome the inhibition when many neighboring cells are active. The last rule is used when the transition-select signal is $\mathrm{ON}$.

\section{CONSTRUCTION OF CELl CIRCUIT}

Using the analog CA model, we developed an analogdigital hybrid circuit for implementing the $\mathrm{BZ}$ operation. As shown in Fig. 4, it consists of neighboring-cell determination, transition-rule, U-generation, and V-generation units. The circuit accepts the output of neighboring cells at the neighboring-cell determination unit and outputs the $U$ from the U-generation unit.

In the V-generation unit, voltage $V_{v}$ between the capacitance $C_{v}$ and the ground represents the value of $V . V_{v}$ increases or decreases with current source $I_{0}$ whether $U=0$ or 1. If $V_{v}$ is larger than the output of the transition-rule unit, $V_{t r}$, the output of the U-generation unit is $U=0$, otherwise $U=1$.

The outputs of the neighboring-cell determination unit, $N_{1}$ and $N_{2}$, represent the number of active neighboring cells. When one or more neighboring cells are active, $N_{1}=1$, otherwise $N_{1}=0$. When two or more neighboring cells are active, $N_{2}=1$, otherwise $N_{2}=0$.

The inputs of the transition-rule unit, $V_{m}$ and $V_{t h}$, represent the mode-select signal and transition-select signal, respectively. The cell operates in oscillatory mode when $V_{m}=1$ and operates in excitatory mode when $V_{m}=0$. When $V_{t h}=0$, the last rule ( $U$ turns active even though $V>0$ ) is used.

The threshold voltages, $V_{\max }$ and $V_{r}$, were determined using the proportion of the capacitances of the transition-rule unit. In the proportion of capacitances illustrated in Fig. 4, $V_{\max }=7 V_{d d} / 10$, and $V_{r}=2 V_{d d} / 10$. 


\section{Simulation RESUlts}

Arranging the cell circuits into a $21 \times 21$ matrix, we designed the network circuit and tested its operation by SPICE simulation, assuming a 1.6- $\mu \mathrm{m}$ double-poly CMOS process.

Figure 5 shows an example operation of the circuit in excitatory mode $\left(V_{m}=0, V_{t h}=0, C_{v}=5 \mathrm{pF}, C=0.1 \mathrm{pF}\right.$, $\left.I_{0}=1.5 \mathrm{~mA}, V_{d d}=5 \mathrm{~V}\right)$. Each cell state is represented in grayscale ( $V=0$, black, $V=V_{\max }$, white). In the circuit's initial state, the active and refractory cells were laid side-by-side. Because the refractory cells inhibited the inactivated cells adjacent to them, those inactivated cells could not turn active. In contrast, the inactivated cells adjacent to the active ones entered an active, refractory, and inactive period in rotation. When the inactive cells were in an active or refractory period, the tip of the white bar (refractory cells) rotated inward, resulting in the generation of the spiral patterns typically observed in the BZ reaction.

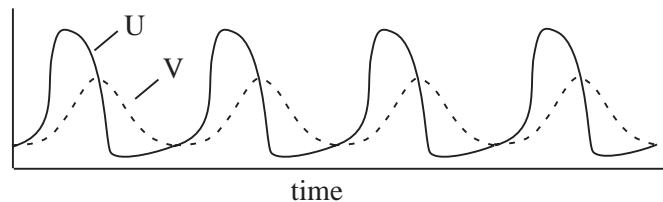

(a) oscillatory behavior

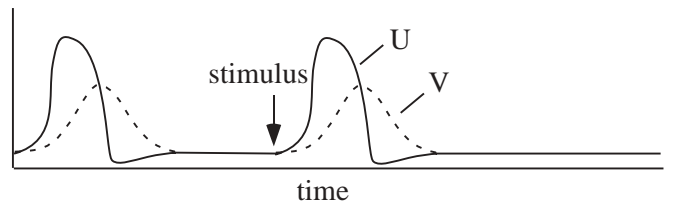

(b) excitatory behavior

Fig. 1 Transient responses of Oregonator

\section{Fig. 1 Transient responses of Oregonator}

\section{CONCLUSION}

We described a continuous-discrete hybrid model of cellular-automaton processing that mimics the BelousovZhabotinsky reaction and a unit-cell circuit that implements the model. A SPICE simulation showed that the proposed circuit can produce the typical spatiotemporal patterns observed in a $\mathrm{BZ}$ reaction.

\section{REFERENCES}

[1] V. Guntik and A. Chandrakasan, 'Active GHz lock network using distributed PLLs'. ISSCC Dig. Tech. Papers, San Francisco, USA. February 2000. pp. 174-175.

[2] G. Nicolis and I. Prigogine, Self-organization in Nonequilibrium Systems - From Dissipative Structures to Order through Fluctuations. John Wiley \& Sons, Inc., 1977.

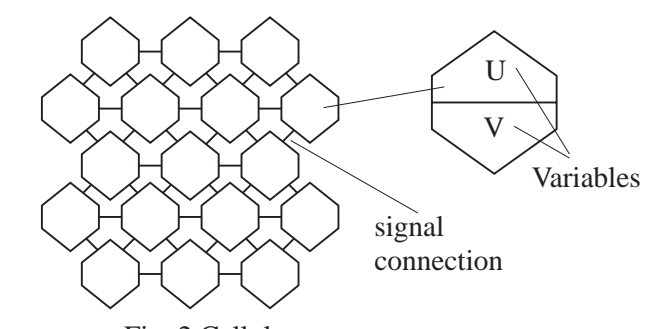

Fig. 2 Cellular automaton

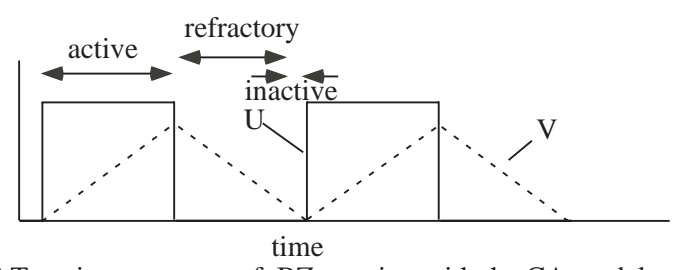

Fig. 3 Transient response of $\mathrm{BZ}$ reaction with the CA model

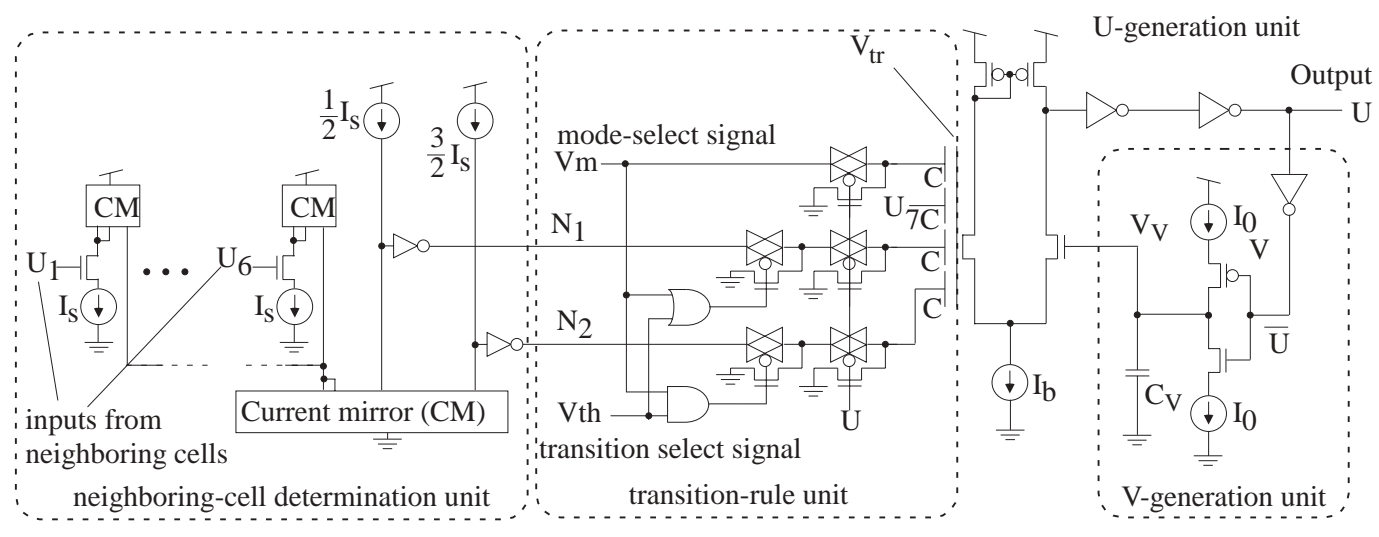

Fig. 4 Cell circuit for RD chip imitated BZ reaction

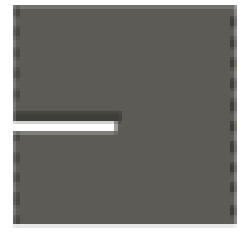

(a) initial pattern

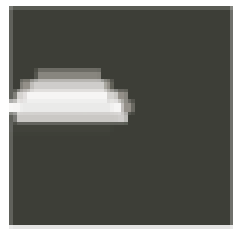

(b) $\mathrm{t}=10 \mu \mathrm{s}$

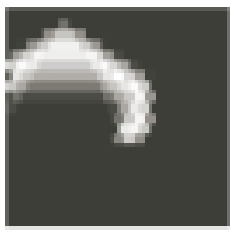

(c) $\mathrm{t}=20 \mu \mathrm{s}$

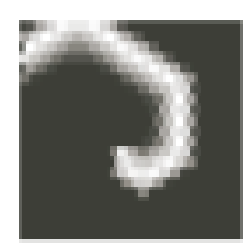

(d) $\mathrm{t}=30 \mu \mathrm{s}$

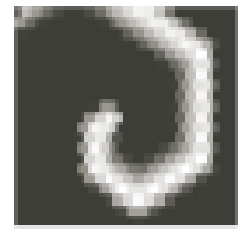

(e) $\mathrm{t}=40 \mu \mathrm{s}$

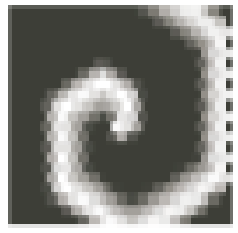

(f) $\mathrm{t}=50 \mu \mathrm{s}$

Fig. 5 Excitatory operations in the network circuit 